\title{
A radioenhancing nanoparticle mediated immunoradiation improves survival and generates long-term antitumor immune memory in an anti-PD1-resistant murine lung cancer model
}

Yun Hu${ }^{1}$, Sébastien Paris ${ }^{2}$, Hampartsoum Barsoumian ${ }^{1}$, Chike O. Abana ${ }^{1}$, Kewen He ${ }^{1,3}$, Duygu Sezen 1,4, Mark Wasley ${ }^{1}$, Fatemeh Masrorpour ${ }^{1}$, Dawei Chen ${ }^{3}$, Liangpeng Yang ${ }^{1}$, Joe D. Dunn ${ }^{1}$, Saumil Gandhi', Quynh-Nhu Nguyen ${ }^{1}$, Maria Angelica Cortez ${ }^{1}$ and James W. Welsh ${ }^{1 *}$

\begin{abstract}
Background: Combining radiotherapy with PD1 blockade has had impressive antitumor effects in preclinical models of metastatic lung cancer, although anti-PD1 resistance remains problematic. Here, we report results from a triplecombination therapy in which NBTXR3, a clinically approved nanoparticle radioenhancer, is combined with high-dose radiation (HDXRT) to a primary tumor plus low-dose radiation (LDXRT) to a secondary tumor along with checkpoint blockade in a mouse model of anti-PD1-resistant metastatic lung cancer.

Methods: Mice were inoculated with 344SQR cells in the right legs on day 0 (primary tumor) and the left legs on day 3 (secondary tumor). Immune checkpoint inhibitors (ICIs), including anti-PD1 (200 $\mu \mathrm{g})$ and anti-CTLA4 $(100 \mu \mathrm{g})$ were given intraperitoneally. Primary tumors were injected with NBTXR3 on day 6 and irradiated with 12-Gy (HDXRT) on days 7, 8, and 9; secondary tumors were irradiated with 1-Gy (LDXRT) on days 12 and 13. The survivor mice at day 178 were rechallenged with 344 SQR cells and tumor growth monitored thereafter.

Results: NBTXR3 + HDXRT + LDXRT + ICls had significant antitumor effects against both primary and secondary tumors, improving the survival rate from 0 to $50 \%$. Immune profiling of the secondary tumors revealed that NBTXR3 + HDXRT + LDXRT increased CD8 T-cell infiltration and decreased the number of regulatory T (Treg) cells. Finally, none of the re-challenged mice developed tumors, and they had higher percentages of CD4 memory T cells and CD4 and CD8 T cells in both blood and spleen relative to untreated mice.
\end{abstract}

Conclusions: NBTXR3 nanoparticle in combination with radioimmunotherapy significantly improves anti-PD1 resistant lung tumor control via promoting antitumor immune response.

Keywords: Nanoparticle, Metastatic lung cancer, Radioimmunotherapy, Checkpoint blockade, Immune memory, NBTXR3, Radiation enhancer, Radiotherapy

\footnotetext{
*Correspondence: jwelsh@mdanderson.org

1 Department of Radiation Oncology, The University of Texas MD

Anderson Cancer Center, 6565 MD Anderson Boulevard, Houston, TX

77030, USA

Full list of author information is available at the end of the article
}

(c) The Author(s) 2021. Open Access This article is licensed under a Creative Commons Attribution 4.0 International License, which permits use, sharing, adaptation, distribution and reproduction in any medium or format, as long as you give appropriate credit to the original author(s) and the source, provide a link to the Creative Commons licence, and indicate if changes were made. The images or other third party material in this article are included in the article's Creative Commons licence, unless indicated otherwise in a credit line to the material. If material is not included in the article's Creative Commons licence and your intended use is not permitted by statutory regulation or exceeds the permitted use, you will need to obtain permission directly from the copyright holder. To view a copy of this licence, visit http://creativecommons.org/licenses/by/4.0/. The Creative Commons Public Domain Dedication waiver (http://creativeco mmons.org/publicdomain/zero/1.0/) applies to the data made available in this article, unless otherwise stated in a credit line to the data. 


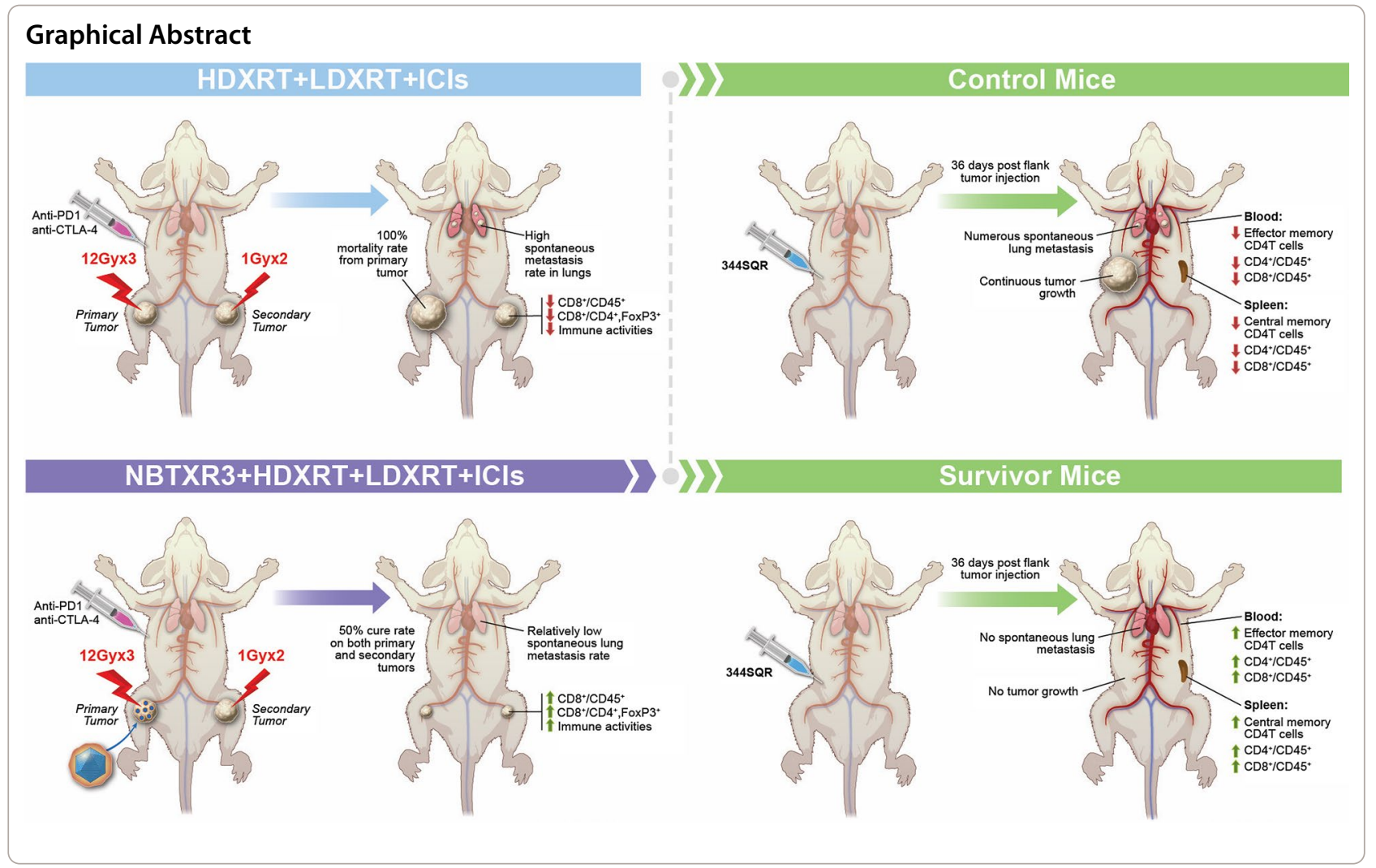

\section{Introduction}

Lung cancer accounts for nearly $25 \%$ of all cancer deaths worldwide [1], and the 5-year survival rates for metastasized lung cancer is only 5\% [2]. In many cases, lung cancer cells have already metastasized at the time of diagnosis, and most patients die of metastatic disease [3]. Therefore, it is critical to develop therapies for effectively treating metastatic lung cancer. Radiation therapy has traditionally been considered a means of local tumor control through its direct effects on cancer cells; however, radiation also has systemic antitumor effects thought to be mediated by the immune system [4-6]. We previously found that delivering high radiation doses to primary tumors in combination with immune checkpoint blockade in a mouse model of metastatic lung cancer could induce potent systemic antitumor immune responses; we further showed that the addition of low doses of radiation to secondary tumors could modulate the immune microenvironment of those tumors [7]. However, this high and low dose radiation strategy was only evaluated in an anti-PD1 sensitive mouse model. It would be of great significance to validate it in an anti-PD1 resistant model, since most of the cancer patients are insensitive to anti-PD1 treatment. In addition, reasoning that augmenting and optimizing these local and systemic immune effects would enhance the effectiveness of this strategy, we explored the effects of adding NBTXR3, a hafnium oxide radioenhancing nanoparticle, to this dualradiation-dose plus checkpoint blockade therapy in a mouse model of anti-PD1 resistant metastatic lung cancer. NBTXR3, which has been approved for the treatment of localized sarcoma [8], increases radiation-mediated damage to cancer cells and promotes activation of the STING pathway $[9,10]$, both of which could contribute to immune priming. We further found that the addition of NBTXR3 to radiation-plus-checkpoint blockade therapy also promoted abscopal effects in the metastatic lung cancer model [11]. However, that combination of localized radiation, NBTXR3, and immunotherapy did not completely eradicate the tumors. Given the potential immune-enhancing capacity of NBTXR3, we hypothesized that integrating these nanoparticles with a highdose-plus-low-dose radiation strategy $\left(\right.$ Radscopal $\left.^{\mathrm{TM}}\right)$, with dual immune checkpoint blockade, would enhance survival, promote systemic antitumor immune responses, and eliminate metastases in this mouse model.

\section{Materials and methods Materials}

The NBTXR3 nanoparticles were provided by Nanobiotix and were kept at room temperature in a dark environment. The mouse anti-PD1 antibody ( $\alpha \mathrm{PD} 1)$ 
(mPD1-4H2-mg1-D265aA) was kindly provided by Bristol-Myers Squibb. The mouse anti-CTLA4 antibody $(\alpha C T L A 4)$ (cat. \#BP0164) was purchased from BioXCell.

\section{Cell line and culture conditions}

The anti-PD1-resistant lung cancer cell line 344SQR, created as described elsewhere [12], was used for all experiments. The $344 \mathrm{SQR}$ cells were cultured in RPMI-1640 medium supplemented with $10 \%$ fetal bovine serum and penicillin/streptomycin and incubated at $37{ }^{\circ} \mathrm{C}$ in a $5 \%$ $\mathrm{CO}_{2}$ atmosphere.

\section{Tumor inoculation and treatment}

Mice in this study were 8- to 12 -week-old $129 / \mathrm{SvEv}$ syngeneic females, purchased from Taconic Biosciences. The $344 S Q R$ cells $\left[5 \times 10^{4}\right.$ in $100 \mu \mathrm{L}$ phosphate-buffered saline (PBS)] were subcutaneously injected into the right legs of the mice on day 0 [to create the "primary" tumor, to be treated with high-dose radiation (HDXRT)] and into the left legs on day 3 [to form the "secondary" tumor, to be treated with low-dose radiation (LDXRT)]. Tumors were measured with digital calipers at least twice a week starting from day 6 , and the tumor volumes were calculated as $\mathrm{V}=0.5 \times$ width $^{2} \times$ length. All mice were given intraperitoneal injections of immune checkpoint inhibitors (ICIs), including anti-PD1 (200 $\mu \mathrm{g} /$ mouse) plus antiCTLA4 (100 $\mu \mathrm{g} / \mathrm{mouse})$ on days $4,7,10$, and 13 , and the anti-PD1 (200 $\mu \mathrm{g} /$ mouse $)$ was continued once a week from day 20 until day 65 . The treatment schedule is shown in Fig. 1A. The five treatment groups were as follows: untreated (control), HDXRT + ICIs, HDXRT + LDXRT + ICIs, NBTXR3 + HDXRT + ICIs, and NBTXR3 + HDXRT + LDXRT + ICIs, with treatment conditions defined as follows. As described in a previous study [11], primary tumors of the HDXRT groups were treated with 3 fractions of 12 Gy each, delivered with an X-RAD $225 \mathrm{Cx}$ small-animal irradiator on days 7, 8, and 9 (total dose 36 Gy). The secondary tumors of the LDXRT groups were treated with 2 fractions of 1 Gy each, also delivered with an X-RAD 225Cx small-animal irradiator on day 12 and 13 (total dose 2 Gy). The dose was delivered with 2 opposing beams from anteroposterior and posteroanterior positions and a $15-\mathrm{mm}$ circular collimator. Dosimetric measurements and treatment planning were done with a MATLAB-based system developed by our in-house radiation physics team at MD Anderson Cancer Center. All collimators were commissioned by Precision XRay Corporation at the time of installation, and routine output checks were done with an ion chamber to ensure that the outputs had not changed and that the treatment plans continued to be accurate. The NBTXR3 groups received intratumoral injection of NBTXR3 $(60.8 \mathrm{mg} /$ $\mathrm{mL}$ ) in $5 \%$ glucose to $25 \%$ of the tumor volume on day
6. Mice were euthanized when any tumor (primary or secondary) reached $14 \mathrm{~mm}$ in any dimension. All animal procedures followed the guidelines of the Institutional Animal Care and Use Committee at MD Anderson Cancer Center.

\section{Tumor rechallenge}

Mice from the NBTXR3 + HDXRT + LDXRT + ICIs group that were still alive at day 178 , and four untreated, 34- to 38-week-old control mice, were given subcutaneous injections of $5 \times 10^{4} 344$ SQR cells in $100 \mu \mathrm{L}$ PBS in the right flank. No further treatment was given. Tumor growth was monitored until the tumors reached $14 \mathrm{~mm}$ in longest diameter. All mice were euthanized 36 days after tumor inoculation, and lungs, spleen, and blood samples were harvested to count the numbers of lung metastases and to obtain profiles of CD4 and CD8 memory $\mathrm{T}$ cells.

\section{Tumor processing}

Secondary tumors were harvested on day 19 for NanoString analyses, and on day 16 for flow cytometry analysis. Tumor tissues were cut into small pieces and digested with $250 \mu \mathrm{g} / \mathrm{mL}$ of Liberase (Roche, cat. \#05401127001) and $20 \mu \mathrm{g} / \mathrm{mL}$ DNAse (Sigma-Aldrich, cat. \#4716728001) at $37^{\circ} \mathrm{C}$ for $30 \mathrm{~min}$. The digestion process was stopped with $1 \mathrm{~mL}$ fetal bovine serum and the samples were filtered. Tumor-infiltrating lymphocytes (TILs) were enriched by using Histopaque 1077 (cat. \#10771, Sigma-Aldrich). RNA was extracted by using a RNeasy Mini Kit (QIAGEN, cat. \#74106) according to the manufacturer's protocol, and the RNA was used for NanoString analysis as described below.

\section{NanoString analysis of immune-related genes}

At least $50 \mathrm{ng}$ of the RNA extracted from each secondary tumor was analyzed with an nCounter PanCancer Immune Profiling Panel and an nCounter MAX Analysis System (both from NanoString Technologies, Seattle, WA, USA) according to the manufacturer's instructions; data were processed with the PanCancer Immune Profiling Advanced Analysis Module (also from NanoString Technologies). Higher immune scores indicate a greater abundance of immune cells.

\section{Flow cytometry analysis}

For these analyses, secondary tumors were obtained on day 16, weighed, digested with Librase and DNAse, and the numbers of cells were counted with a TC20 Automated Cell Counter (Bio-Rad). Cells were then stained with anti-CD45-APC (cat. \#103112), anti-CD3BV510 (cat. \#100234), anti-CD4-PE (cat. \#100408), and anti-CD8-PercpCy5.5 (cat. \#100734) (all from 
A

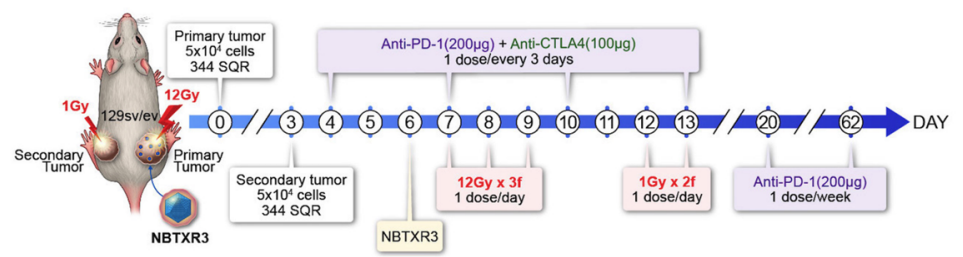

B

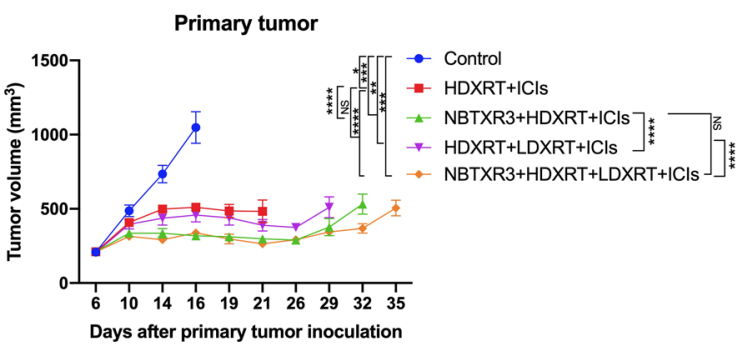

D

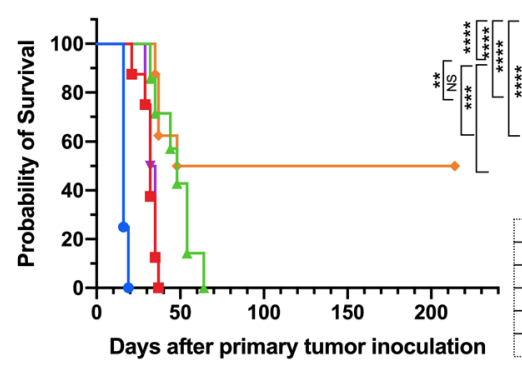

- Control

$\rightarrow$ HDXRT+ICls

- NBTXR3+HDXRT+ICls

$\rightarrow$ HDXRT+LDXRT+ICls

- NBTXR3+HDXRT+LDXRT+ICls

\begin{tabular}{|l|l|} 
& Median survival \\
\hline Control & 16 \\
\hline HDXRT+|Cls & 32 \\
\hline NBTXR3+HDXRT+|Cls & 48 \\
\hline HDXRT+LDXRT+ICls & 33.5 \\
\hline NBTXR3+HDXRT+LDXRT+ICls & 131 \\
\hline
\end{tabular}

Secondary tumor

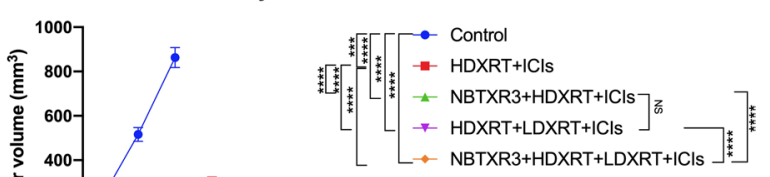

F
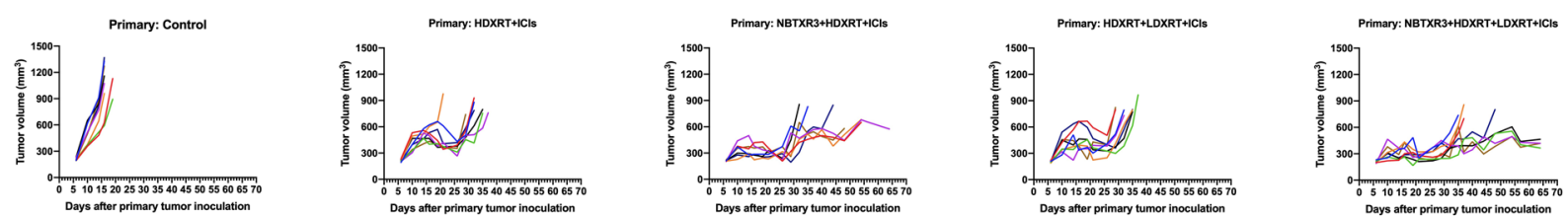

G
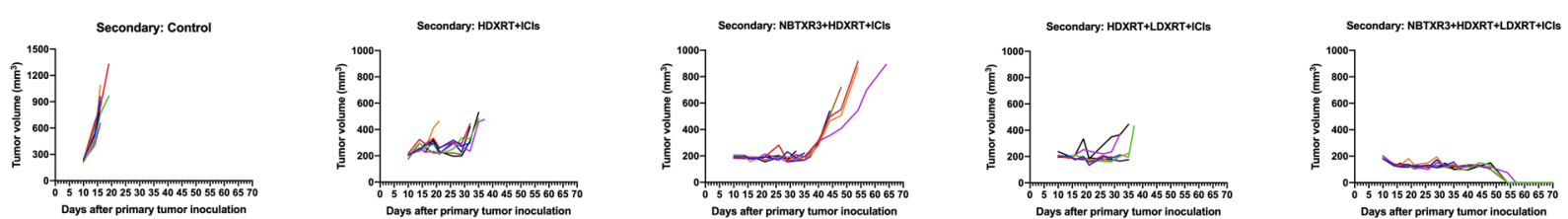

Fig. 1 Treatment outcomes after therapy with NBTXR3, high- and low-dose radiotherapy, and immunotherapy. A Treatment schema for NBTXR3 given with high-dose and low-dose radiotherapy. Mice were subcutaneously inoculated with $5 \times 10^{4} 344$ SQR cells in the right legs on day 0 (to establish primary tumors) and in the left legs on day 3 (to establish secondary tumors). NBTXR3 was delivered to the primary tumor by intratumoral injection on day 6. Primary tumors were treated with three 12-Gy fractions on day 7, 8, and 9 (HDXRT). Secondary tumors were irradiated with

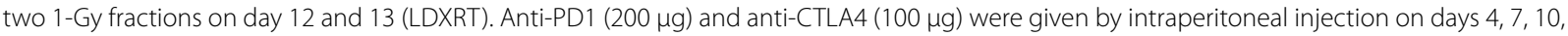
and 13, and anti-PD1 treatment was continued once a week from day 20 until day 62. B Changes in primary tumor volumes over time. C Changes in secondary tumor volumes over time. Data are shown as means for each treatment group ( $n=7$ or 8 ), with bars indicating standard error of the mean. D Survival rates and median survival times for each treatment group. E Number of spontaneous lung metastases on day 16 in each treatment group. All of the mice were injected with both anti-PD1 and anti-CTLA4, and all mice were euthanized when the any tumor exceeded $14 \mathrm{~mm}$ in diameter. $\mathbf{F}$ Individual primary tumor volumes for each treatment group. G Individual secondary tumor volumes for each treatment group. Data are expressed as means \pm standard error of the mean (SEM). $P<0.05$ was considered statistically significant. ${ }^{*} P<0.05$, ${ }^{* *} P<0.01$, ${ }^{* * *} P$ $<0.001,{ }^{* * *} P<0.0001$, NS not significant. HDXRT high-dose radiotherapy; LDXRT low-dose radiotherapy; ICIs immune checkpoint inhibitors 
BioLegend). For intracellular staining of the Treg marker FOXP3, cells were fixed and permeabilized according to the manufacturer's instructions (BioLegend) and stained with anti-Foxp3-Alexa 488 (cat. \#126406).

Splenocytes and blood samples from the tumor rechallenge experiment were stained with anti-CD45Pacific Blue (cat. \#103126), anti-CD4-APC-Fire 750 (cat. \#100460), anti-CD8-PercpCy5.5 (cat. \#100734), antiCD62L-PE-Cy7 (cat. \#104418), and anti-CD44-APC (cat. \#103012) (all from BioLegend). Samples were analyzed with a Gallios Flow Cytometer (Beckman Coulter) with Kaluza software Version 2.1.

\section{Analysis of numbers of lung metastases}

Lungs were harvested either on day 16 , or, in the tumor rechallenge experiment, from both the control group and the NBTXR3 + HDXRT + LDXRT + ICIs group on day 36 after the rechallenge. The extracted lungs were stored in Bouin's fixative solution (Polysciences Inc., cat. \#16045-1) for 3 days, after which lung metastatic nodules were counted.

\section{TCR repertoire analysis}

Four groups of 4 mice were treated with HDXRT + ICIs, HDXRT + LDXRT + ICIs, NBTXR3 + HDXRT + ICIs, and NBTXR3 + HDXRT + LDXRT + ICIs as described above. Total RNA was extracted from secondary tumors on day 19. TCR sequencing was performed by a method described in a previous study [11].

\section{Statistical analyses}

All statistical analyses were done with Prism 8.0 (GraphPad Software). Tumor growth curves were compared by two-way analysis of variance and were expressed as mean tumor volume \pm standard error of the mean (SEM). Mouse survival rates were analyzed with the KaplanMeier method, and estimates were compared with logrank tests. The Nanostring data of Total TILs score and CD8 T cells score were analyzed with one-way ANOVA, and the Treg score and dendritic cell score were analyzed with Kruskal-Wallis test. All other data were analyzed with two-tailed $t$ tests and expressed as mean value \pm SEM. $P$ values of $<0.05$ were considered to indicate statistically significant differences.

\section{Results}

\section{NBTXR3 nanoparticle in combination with high} and low-dose radiation improves tumor control and reduces the number of spontaneous lung metastases Mice that received radiation had both primary and secondary tumors that were both smaller and grew more slowly than in the control mice (Fig. 1B). Further, consistent with our previous findings [11], primary tumors treated with both NBTXR3 and radiation grew more slowly than those treated with radiation alone $(P$ $<0.0001$; Fig. 1B). Moreover, HDXRT to the primary tumor delayed the growth of the secondary tumors relative to the control $(P<0.0001$; Fig. $1 C)$, thereby indicating an abscopal effect; giving NBTXR3 with HDXRT + ICIs further slowed growth of the secondary tumor relative to HDXRT + ICIs alone $(P<0.0001$; Fig. 1C). The addition of LDXRT also delayed secondary tumor growth (HDXRT + LDXRT + ICIs vs. HDXRT + ICIs, $P<0.0001 ;$ NBXR3 + HDXRT + LDXRT + ICIs vs. NBTXR3 + HDXRT + ICIs, $P$ $<0.0001$; Fig. 1C). As shown in Additional file 1: Fig. S1, although NBTXR3 + HDXRT + LDXRT considerably reduced the growth of the primary tumor, it had little effect over the secondary tumor. Notably, the combination therapy (NBTXR3 + HDXRT + LDXRT + ICIs) completely eradicated both the primary and secondary tumors in 4 of the 8 of the mice in that treatment group. The results indicate that ICIs are essential for controlling the secondary tumors.

The addition of NBTXR3 was also beneficial in terms of extending median survival times, from 16 days for the control group to 32 days for HDXRT + ICIs only, 33.5 days for HDXRT + LDXRT + ICIs, 48 days for NBTXR3 + HDXRT + ICIs, and 131 days for the NBTXR3 + HDXRT + LDXRT + ICIs group (Fig. 1D). In terms of tumor-related survival, NBTXR3 may also have improved rates of death from primary and secondary tumors: all of the mice in the control group $(\mathrm{n}=8)$, the HDXRT + ICIs group $(\mathrm{n}=8)$, and the HDXRT + LDXRT + ICIs group $(n=8)$ were expired due to the growth of the primary tumor, i.e., the primary tumor exceeded $14 \mathrm{~mm}$ in greatest diameter; but only 3 of 7 mice in the NBTXR3 + HDXRT + ICIs group and 4 of 8 mice in the NBTXR3 + HDXRT + LDXRT + ICIs groups died from the primary tumor (Fig. 1F). In terms of death from the secondary tumors, 4 of the 7 mice in the NBTXR3 + HDXRT + ICIs group died, but none of the 8 mice in the NBTXR3 + HDXRT + LDXRT + ICIs group expired because of growth of the secondary tumor (Fig. 1G). Finally, the combination therapy also led to fewer spontaneous lung metastases: the NBTXR3 + HDXRT + LDXRT + ICIs group had $6 \pm 2$ lung metastases as compared with $12 \pm 2$ for the HDXRT + LDXRT + ICIs group, $14 \pm 1$ for the NBTR3 + HDXRT + ICIs group, $21 \pm 3$ for the HDXRT + ICIs group, and $35 \pm 4$ for the control group (Fig. 1E). These results suggest that the combination therapy was also effective in reducing the number of spontaneous lung metastases in this model. 
NBTXR3 + HDXRT + LDXRT + ICls modulates expression of immune-related antitumor genes

Next, we investigated how NBTXR3 and LDXRT affect immune-related gene expression patterns (and, theoretically, control of the secondary tumors). NanoString analysis of the RNA in immune cells from the secondary tumors (extracted at day 19) showed that all of the treatment conditions (relative to the control conditions) upregulated the activity of immune pathways involved in adaptive immune response, innate immune response, antigen processing, T-cell function, NK-cell function, and dendritic cell function (Fig. 2A). NBTXR3 + HDXRT + LDXRT + ICIs seemed to have increased the activity of these immune pathways to a greater extent than did HDXRT + LDXRT + ICIs (Fig. 2A), which is consistent with our previous finding that NBTXR3 could upregulate major immune pathways [11]. However, LDXRT to the secondary tumors in this study seemed to suppress the activities of these immune pathways. The comparison of individual gene expression (Fig. 2B) reveals that the low dose radiation on the secondary tumor significantly upregulated expression of the genes, such as Cd8a, Ifngr1, GzmK, Cd86, Cd83, Casp3, etc. The addition of NBTXR3 to high- and lowdose radiation clearly affected expression of genes, such as Gzmb, Cd8a, Itgal, Ccl3, Il1a, etc., which are associated with T-cell and NK-cell function, innate immunity, and adaptive immunity (Fig. 2B). Remarkably, the addition of NBTXR3 to HDXRT + LDXRT + ICIs downregulated Atg5 in the secondary tumors. It has been reported that the knockout of ATG5 polarized macrophages to M1 phenotype, which increased inflammatory response [13]. In addition, NBTXR3 also upregulated Illa expression. IL- $1 \alpha$, which can be expressed by macrophages, has been shown to strongly induce apoptosis $[14,15]$. IL-1 $\alpha$ mediated-anti-tumor immune response may also develop into immunological memory against cancer cells [16]. In terms of numbers of each immune cell type, all of the treatments led to increases in TIL score, dendritic cell score, and CD8 T-cell score relative to the control condition (Fig. 2C). As for numbers of Tregs, the HDXRT + ICIs and NBTXR3 + HDXRT + ICIs groups had more Tregs in the secondary tumor than the control group. In contrast, the numbers of Tregs in the HDXRT + LDXRT + ICIs and NBTXR3 + HDXRT + LDXRT + ICIs groups were similar to that in the control group.

\section{Adding NBTXR3 nanoparticle to high- and low-dose radiation shifted immune cell subpopulations to favor tumor control}

We further confirmed the shift in proportions of the various subtypes of immune cells from the secondary tumors via flow cytometry analysis. None of the various treatments significantly affected the $\mathrm{CD} 4^{+} / \mathrm{CD} 45^{+}$ratio (Fig. $3 \mathrm{~A}$ ) or the Treg $\mathrm{CD}^{+} \mathrm{FoxP3}^{+} / \mathrm{CD} 45^{+}$ratio (Fig. $3 \mathrm{C}$ ). However, NBTXR3 + HDXRT + LDXRT + ICIs increased the percentage of $\mathrm{CD}^{+} \mathrm{T}$ cells (to $5.71 \pm 0.88 \%$ ) over that in the other groups (Fig. 3B). Also, in the secondary tumors, the NBTXR3 + HDXRT + LDXRT + ICIs treatment led to a significantly higher CD8 T cell/ Treg ratio $(2.70 \pm 0.39)$ than the control $(1.16 \pm 0.21)$, HDXRT + LDXRT + ICIs (1.37 \pm 0.29$)$, and NBTXR3 + HDXRT + ICIs $(1.20 \pm 0.28)$ conditions (Fig. 3D). Unexpectedly, the HDXRT + LDXRT + ICIs and NBTXR3 + HDXRT + LDXRT + ICIs groups had less dense CD4 T cells than in the HDXRT + ICIs and NBTXR3 + HDXRT + ICIs groups, respectively (Fig. 3E), suggesting that LDXRT negatively affects the infiltration of CD4 $\mathrm{T}$ cells into the secondary tumors. With regard to $\mathrm{CD} 8^{+}$ T cell density, the NBTXR3 + HDXRT + ICIs condition led to the highest density $(2.55 \pm 0.56 \mathrm{million} / \mathrm{g})$, followed by the HDXRT + LDXRT + ICIs (1.46 \pm 0.18 million/g) and NBTXR3 + HDXRT + LDXRT + ICIs $(2.12 \pm 0.31 \mathrm{million} / \mathrm{g})$ condition, and all were higher than in the control condition $(0.85 \pm 0.17$ million $/ \mathrm{g})$ (Fig. 3F). The NBTXR3 + HDXRT + ICIs condition also increased the density of Tregs $(2.43 \pm 0.54 \mathrm{million} / \mathrm{g})$ relative to the control $(0.81 \pm 0.23$ million/g), but the NBTXR3 + HDXRT + LDXRT + ICIs condition decreased the density of Tregs $(0.82 \pm 0.13$ million/g) relative to the NBTXR3 + HDXRT + ICIs group (Fig. 3G). These results suggest that the antitumor effectiveness of NBTXR3 + HDXRT + LDXRT + ICIs could have resulted from enhanced infiltration of CD8 $+\mathrm{T}$ cells and a favorable CD8/Treg ratio.

\section{NBTXR3 nanoparticle, given with immunoradiation, reshapes the TCR $\beta$ repertoire}

We analyzed the TCR repertoire of tumor-infiltrating $\mathrm{T}$ cells within the metastatic tumors for the effect of NBTXR3. Circos plots of TCR variable (V) and joining (J) gene family pairs of productive CDR3 $\beta$ and CDR3 $\alpha$ are shown in Fig. 4A (representative figures from each

\footnotetext{
(See figure on next page.)

Fig. 2 NanoString analysis of immune-gene expression in the secondary tumors. A Heatmap of the activities of various immune pathways. B Changes in gene expression in innate pathway, adaptive pathway, T cell and NK cell function in the NBTXR3 + HDXRT + LDXRT + ICls group relative to the NBTXR3 + HDXRT + ICls group and the HDXRT + LDXRT + ICls group. C Relative scores for total tumor-infiltrating lymphocytes (TILs), dendritic cells, CD8 T cells, and regulatory T (Treg) cells. Data are expressed as means \pm standard error of the mean (SEM). $P<0.05$ was considered statistically significant. ${ }^{*} P<0.05,{ }^{* *} P<0.01$, ${ }^{* * *} P<0.001$, NS not significant. HDXRT high-dose radiotherapy; LDXRT low-dose radiotherapy; ICls immune checkpoint inhibitors
} 
A

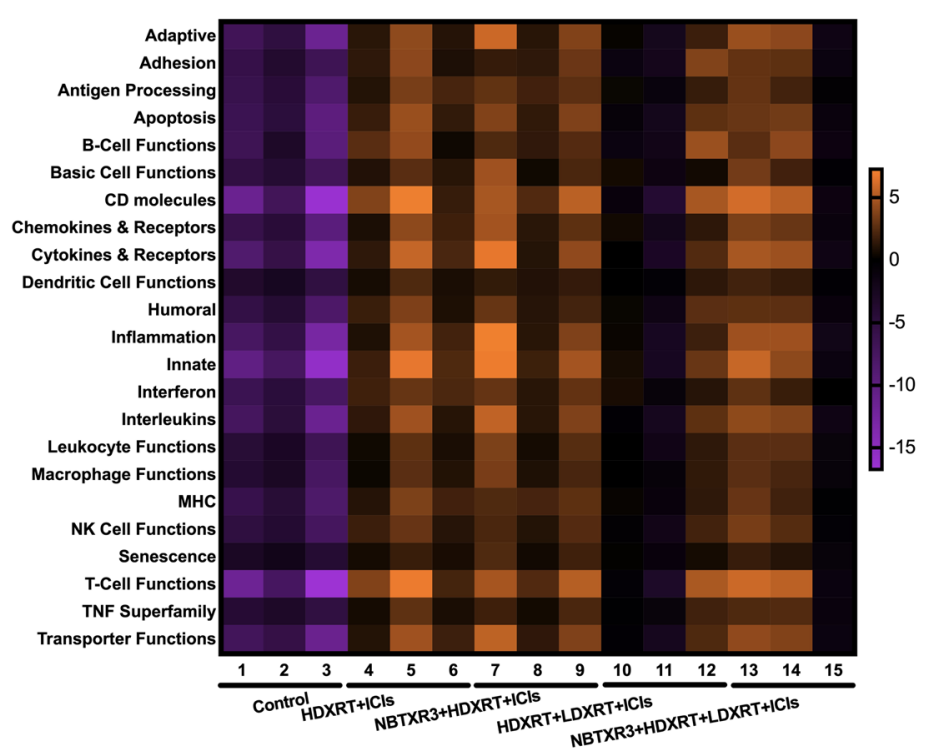

NBTXR3+HDXRT+LDXRT+ICIs vs NBTXR3+HDXRT+ICIs

Innate pathway

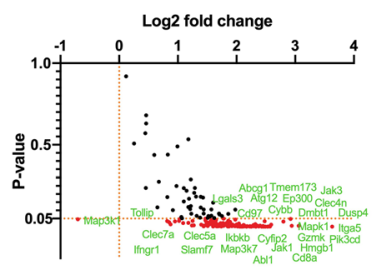

Adaptive pathway

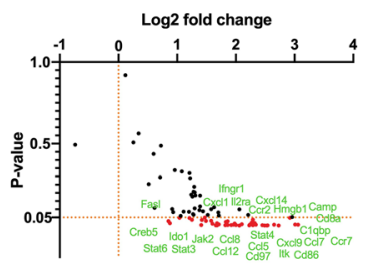

T cell function

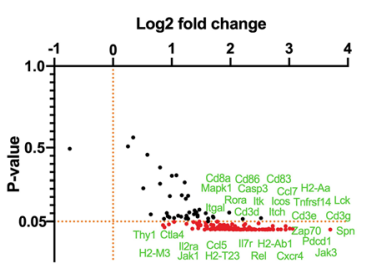

NK cell function

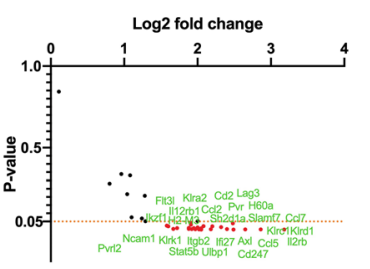

\section{NBTXR3+HDXRT+LDXRT+ICIs vs HDXRT+LDXRT+ICIs}

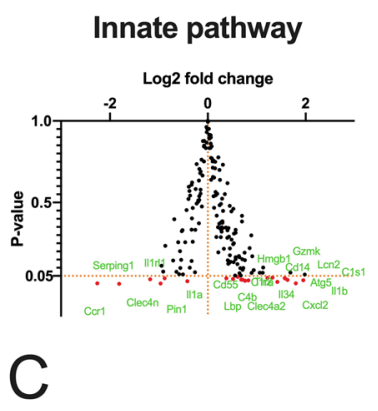

Adaptive pathway
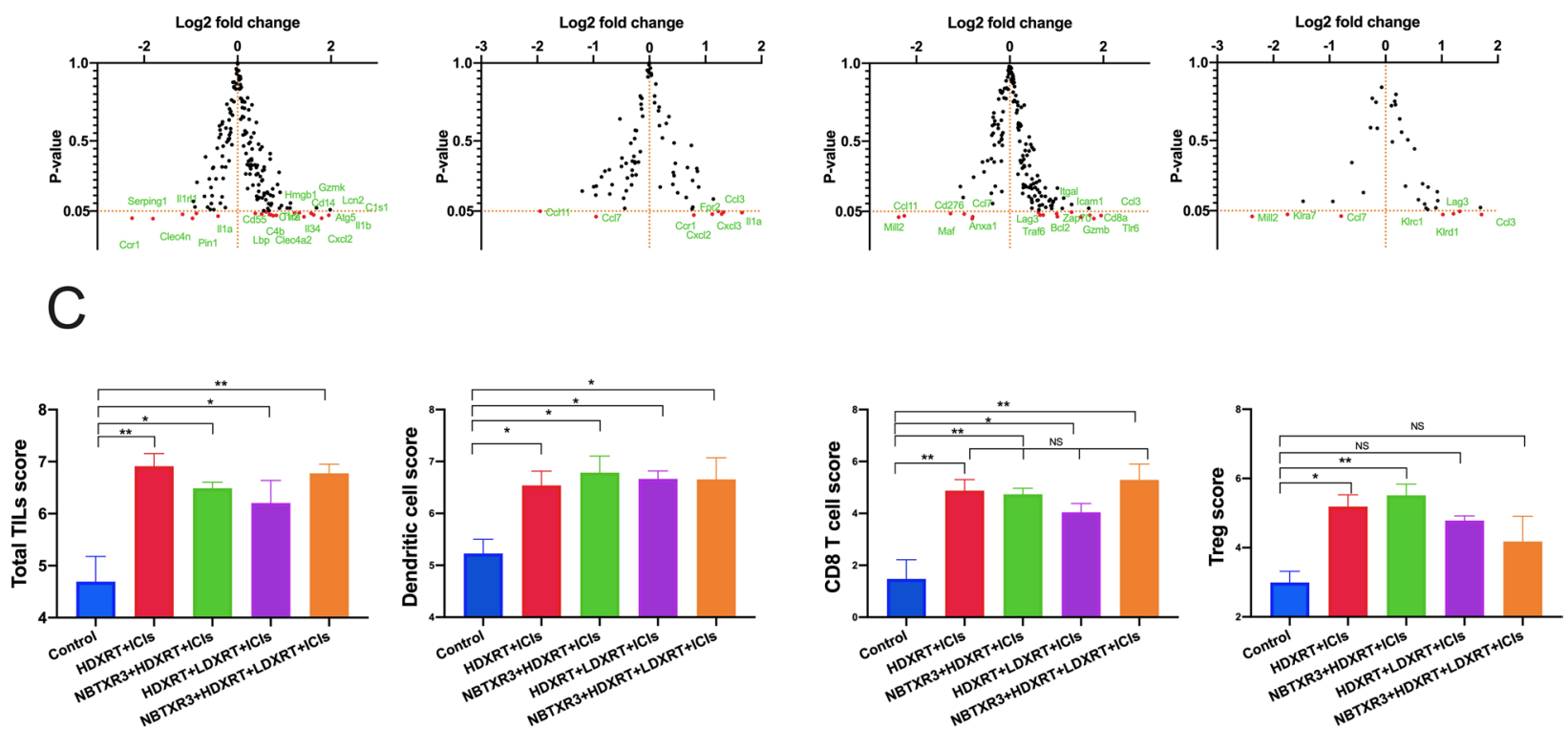

Fig. 2 (See legend on previous page.) 

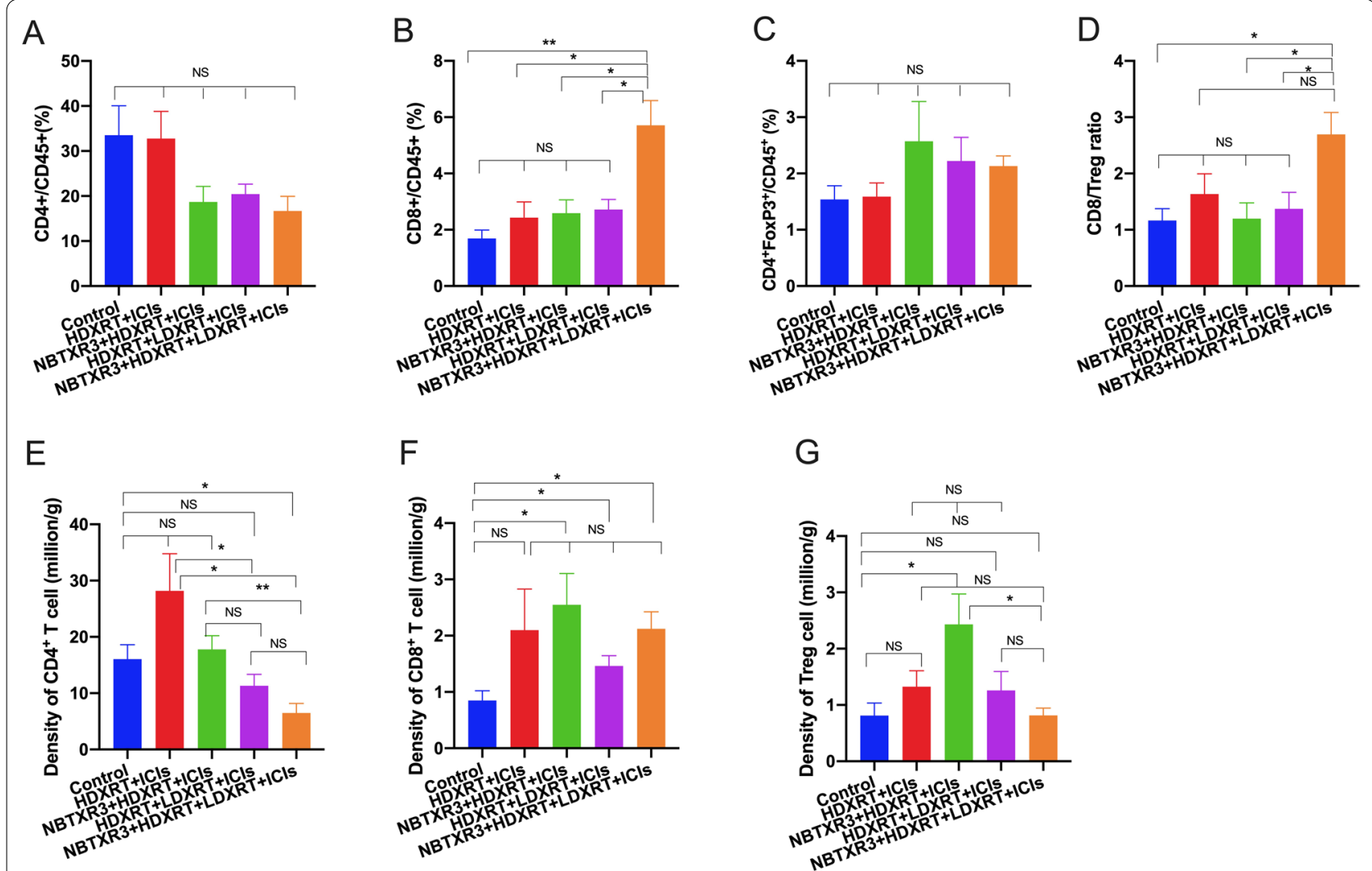

Fig. 3 Flow cytometry analysis of $\mathrm{CD} 4^{+} \mathrm{T}$ cells, $\mathrm{CD} 8^{+} \mathrm{T}$ cells, and regulatory $\mathrm{T}$ cells (Tregs, labeled with $\mathrm{CD}^{+} \mathrm{FoxP}^{+}$) in the secondary tumors, harvested on day $16 . \mathbf{A} C D 4^{+} \mathrm{T}$ cell/CD45 ${ }^{+}$cell ratio. $\mathbf{B} \mathrm{CD} 8^{+} \mathrm{T}$ cell/CD45 $5^{+}$cell ratio. $\mathbf{C}$ Treg $\mathrm{CD} 4^{+} \mathrm{FoxP3} 3^{+}$cell/CD45 ${ }^{+}$cell ratio. $\mathbf{D}$ CD8 ${ }^{+} \mathrm{T}$ cell/ Treg $\mathrm{CD} 4^{+} \mathrm{FoxP}^{+}$cell ratio. E Density of $\mathrm{CD} 4^{+} \mathrm{T}$ cells. $\mathbf{F}$ Density of $\mathrm{CD} 8^{+} \mathrm{T}$ cells. $\mathbf{G}$ Density of Treg $\mathrm{CD} 4^{+}{ }^{\mathrm{FoxP}} 3^{+}$cells. Data are expressed as means \pm standard error of the mean (SEM). $P<0.05$ was considered statistically significant. ${ }^{*} P<0.05,{ }^{* *} P<0.01,{ }^{* * *} P<0.001,{ }^{* * * *} P<0.0001$, NS not significant. HDXRT high-dose radiotherapy; LDXRT low-dose radiotherapy; /Cls immune checkpoint inhibitors

group). To determine whether there was any difference in the diversity of the repertoires due to NBTXR3, we analyzed the normalized Shannon clonality indices between the NBTXR3 groups and their non-NBTXR3 counterparts but observed no differences in diversity for both CDR3 $\beta$ and CDR3 $\alpha$ sequences (Additional file 2: Fig. S2), confirming our previous findings [11]. As a result, we hypothesized that the NBTXR3-induced tumor control that we observed in all NBTXR3-treated mice was likely mediated by tumor-specific $\mathrm{T}$ cells shared among the NBTXR3-treated mice. To test this hypothesis, we used the F measure, which is highly sensitive at grouping or differentiating TCR sequences based on degree of similarity [17], to compare the degree of overlapping sequences shared between the non-NBTXR3-treated groups with the clonotypes shared between the NBTXR3 groups. Among CDR3 $\beta$ s, we observed that NBTXR3 significantly increased the frequencies of shared clonotypes among the mice that received only high dose of radiation (NBTXR3 + HDXRT + ICIs vs. HDXRT + ICIs, $P=0.0476$, Fig. 4B), but it did not significantly increase them among the mice treated with high and low dose $\left(\operatorname{Radscopal}^{\mathrm{TM}}\right)$ radiation (NBTXR3 + HDXRT + LDXRT + ICIs vs. HDXRT + LDXRT + ICIs, $P=0.3095$, Fig. 4B). Furthermore, NBTXR3 increased the number of overlapping CDR3 $\beta$ sequences shared between the "NBTXR3 + HDXRT + ICIs" and "NBTXR3 + HDXRT + LDXRT + ICIs" groups compared to those shared between these same groups in the absence of NBTXR3 $(P<0.0001$, Fig. 4B). None of these observations were made among the CDR3 $\alpha$ however (Fig. 4C), which might be due to the presence of two TCR $\alpha$ per T cell [18]. These higher proportions of overlapping CDR3 $\beta$ sequences with NBTXR3 suggests that NBTXR3 might be inducing the expression of certain nanoparticle-associated epitopes that are recognized by the shared repertoires.

\section{Adding NBTXR3 nanoparticle to high- and low-dose radiation induces robust long-term immune memory}

As noted earlier, 4 of the 8 mice in the NBTXR3 + HDXRT + LDXRT + ICIs survived and showed no evidence of either primary or secondary 


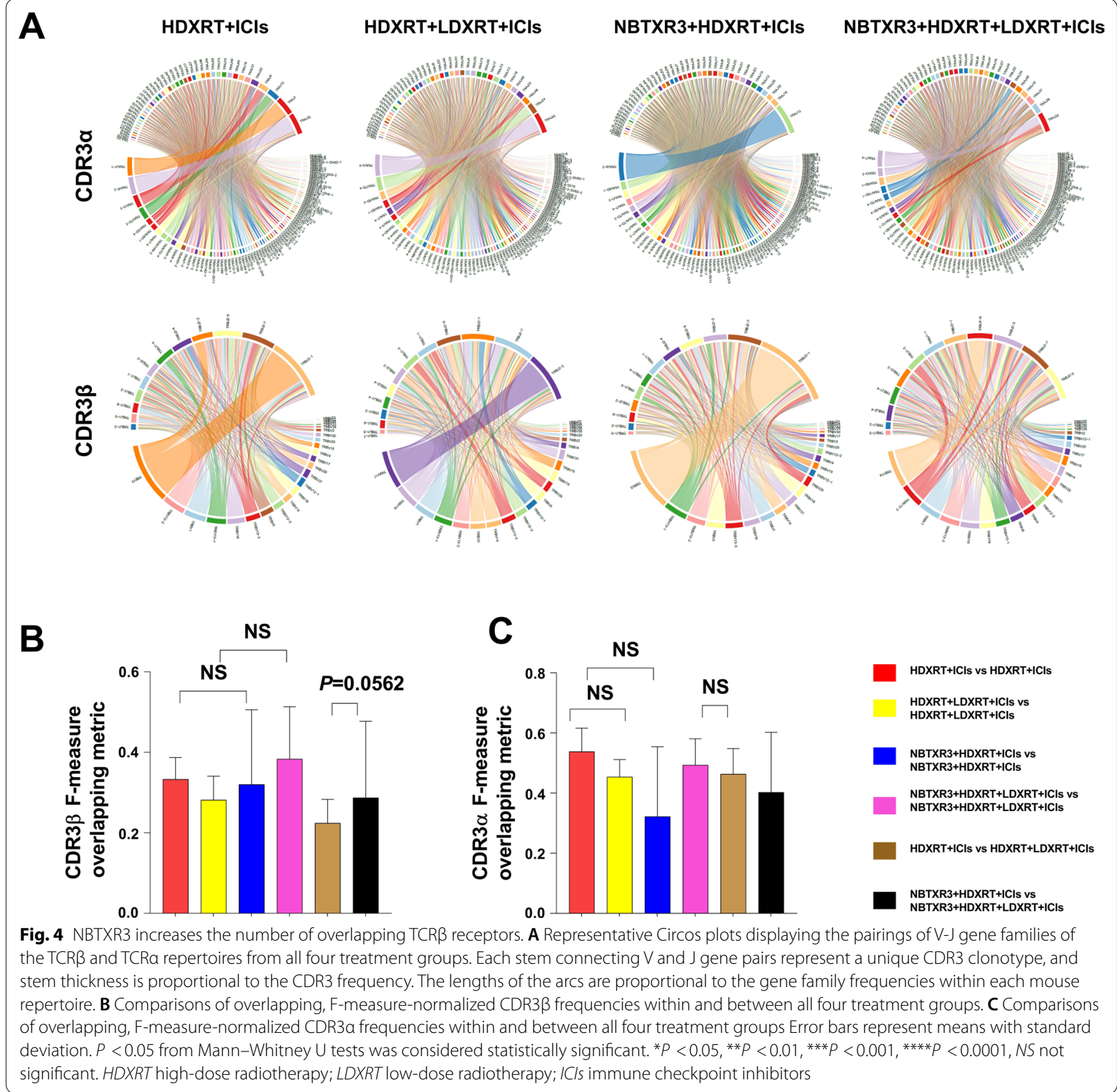

tumors at the end of the first experiment, prompting the question of whether these mice had developed an effective immune memory against the lung cancer cells. To this end, the 4 mice from the NBTXR3 + HDXRT + LDXRT + ICIs group that survived were re-challenged with $344 S Q R$ cells in the right flank at day 178 . Another 4 untreated mice, aged 34-38 weeks (i.e., about the same age as the surviving mice) were also inoculated with 344SQR cells in the right flank to function as a control group. No treatment was given to any of these mice, and tumor growth was monitored for 36 days. Tumors in the control group grew steadily over that period (Fig. 5A), but no tumors were detected in the mice in the NBTXR3 + HDXRT + LDXRT + ICIs group during that same period (Fig. 5A). On day 36 after the rechallenge, lungs were harvested from the mice and lung metastases counted. At that time, mice in the control group had a mean ( \pm SEM) of $19 \pm 7$ lung tumor nodules but the survivors from the NBTXR3 + HDXRT + LDXRT + ICIs had no lung metastases (Fig. 5B). These results suggest that the surviving mice may have developed a systemic antitumor response. 
A

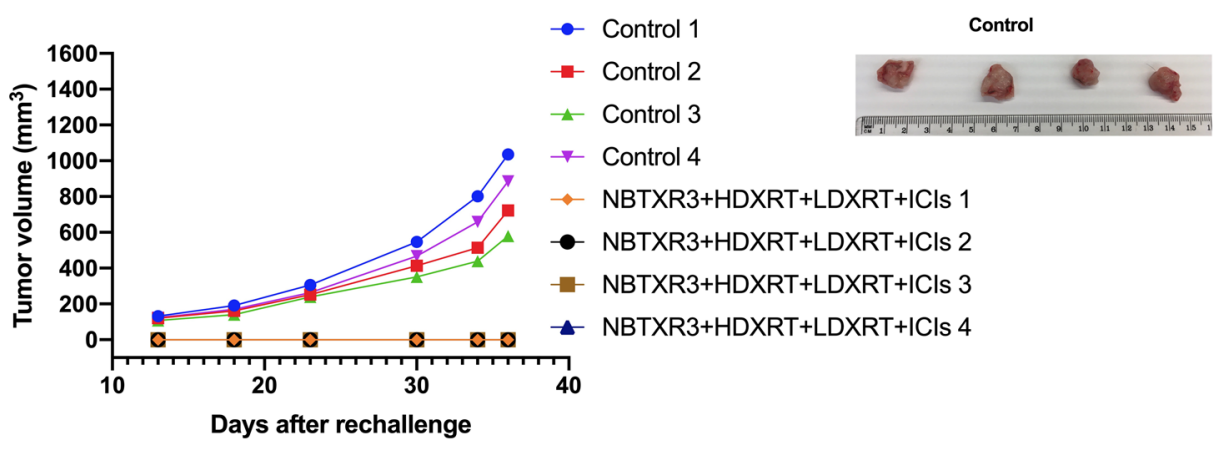

B

\section{Control}
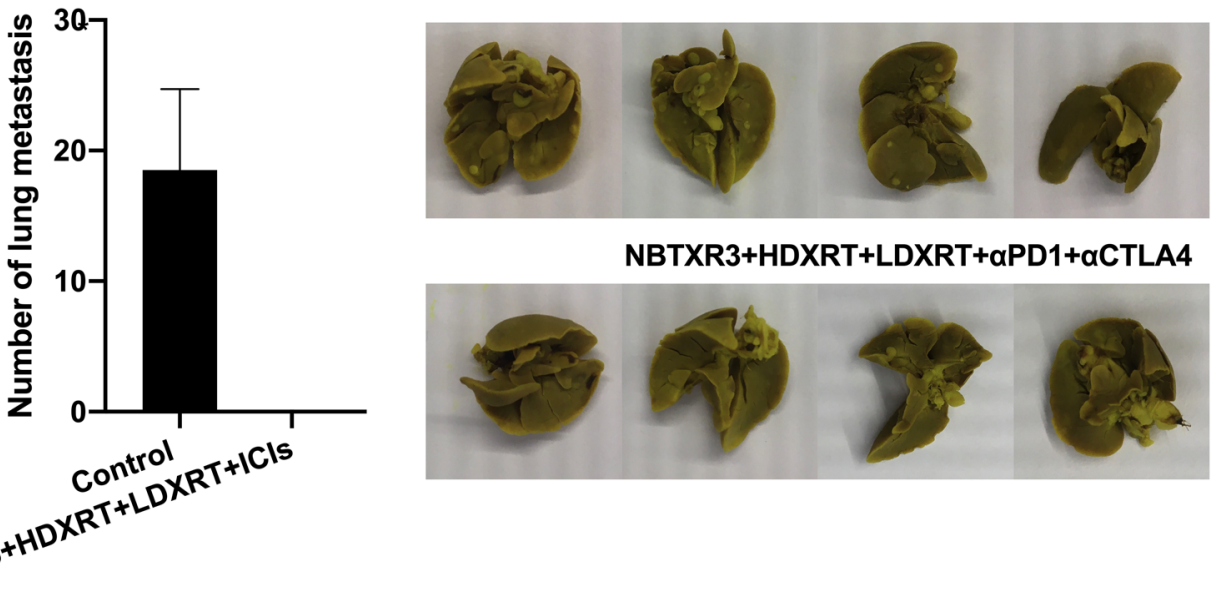

NBTXR3+HDXRT+LDXRT+aPD1+aCTLA4

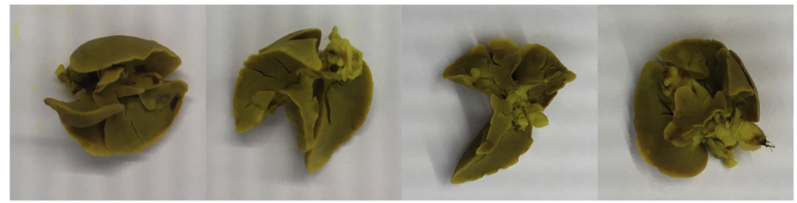

$N B T R R^{3}$

Fig. 5 Tumor growth curves and lung metastases in mice rechallenged with 344SQR cells. A Left, tumor growth curves for each of the surviving mice from the NBTXR3 + HDXRT + LDXRT + ICls treatment group and the four control mice after rechallenge with 344SQR cells in the right flank. Right, images of excised tumors from the control group. B Numbers (left) and images (right) of lung metastases at 36 days after tumor rechallenge. Data are expressed as means \pm standard error of the mean (SEM). $P<0.05$ was considered statistically significant. ${ }^{*} P<0.05$. HDXRT high-dose radiotherapy; LDXRT low-dose radiotherapy; ICls immune checkpoint inhibitors

Finally, to further explore the question of whether immune memory had been evoked in these mice after rechallenge, we used flow cytometry to analyze the proportions of CD4 and CD8 memory cells in spleen and blood samples from those mice at the end of the experiment. The NBTXR3 + HDXRT + LDXRT group had more central memory $\mathrm{CD} 4 \mathrm{~T}$ cells in the spleen than did the control group $(16.1 \pm 0.4 \%$ vs. $9.2 \pm 2.0 \%)(P<0.05$; Fig. 6A), and the NBTXR3 + HDXRT + LDXRT + ICIs mice also had more effector memory CD4 $\mathrm{T}$ cells in the blood than did the controls $(14.5 \pm 1.0 \%$ vs. $7.9 \pm 1.8 \%)(P<0.05$; Fig. $6 \mathrm{~B})$. Percentages of memory CD8 T cells in both spleen and blood were either too low to be measured or were no different between the control and NBTXR3 + HDXRT
+ LDXRT + ICIs groups (Fig. 6A, B). In terms of percentages of CD4/CD45 and CD8/CD45 cells, in the spleen the NBTXR3 + HDXRT + LDXRT mice had higher percentages of CD4 T cells $(30.2 \pm 1.6 \%)$ and CD8 $\mathrm{T}$ cells $(7.6 \pm 0.2 \%)$ than did the control group [CD4 T cells, $23.4 \pm 0.3 \%(P<0.01)$; and CD8 T cells, $3.8 \pm 0.2 \%(\mathrm{P}<0.0001)$ ] (Fig. $6 \mathrm{C})$. In the blood, the NBTXR3 + HDXRT + LDXRT mice also had significantly higher percentages of CD4 T cells $(37.8 \pm 1.3 \%)$ and CD8 T cells $(5.4 \pm 0.7 \%)$ than did the control mice [CD8 T cells, $0.5 \pm 0.2 \%(P<0.01)$ ]; and CD4 T cells $[20.3 \pm 3.2 \%(P<0.001)]$ (Fig. 6D). The higher percentages of both CD4 and CD8 T cells in spleen and blood suggest that mice treated with NBTXR3 + HDXRT + LDXRT + ICIs maintained a long-term adaptive 


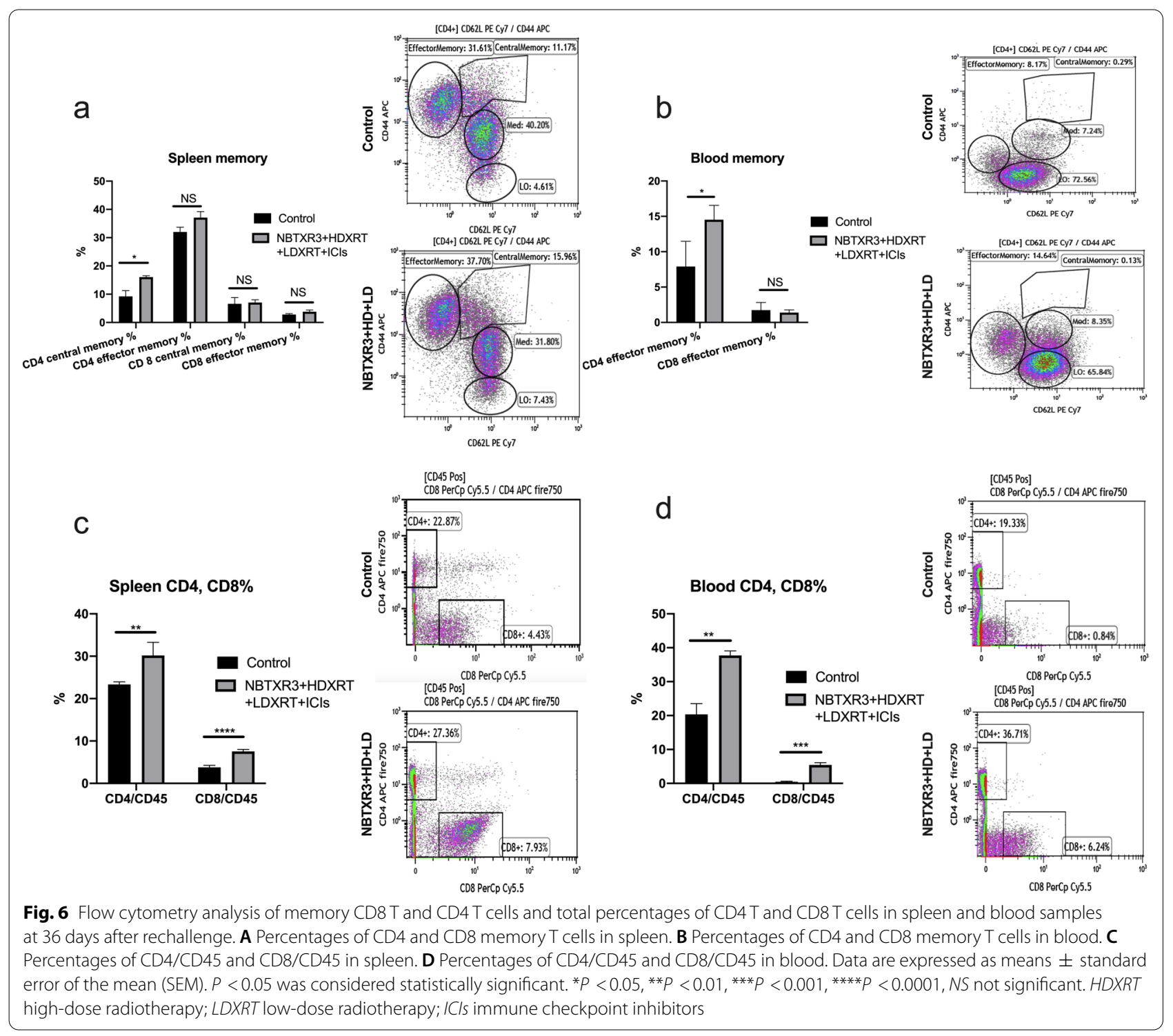

immune response against tumor, which could be responsible for eradicating the re-injected cancer cells.

\section{Discussion}

Outcomes after treatment for metastatic lung cancer depend mainly on effective control of the metastases $[19,20]$. Combination radiation-plus-immune checkpoint inhibitors have emerged as a promising strategy for inducing a systemic antitumor immune response, a key component of effectively treating metastatic cancers [5]. We previously reported a radiotherapy schedule $\left(\right.$ RadScopal $^{\mathrm{TM}}$ ) consisting of HDXRT to primary tumors and simultaneous LDXRT to secondary tumors that was developed to induce such an antitumor immune response with the goal of treating secondary (metastatic) tumors [7]. The HDXRT acts to prime the immune system at the primary tumor, and the LDXRT facilitates infiltration of anti-tumor lymphocytes and favors M1 macrophage polarization. In the current study, the integration of NBTXR3 into the high- and low-dose radiation strategy improved control of both the primary and secondary tumors as well as extending survival. Presumably the effects of NBTXR3 on the primary tumor result from its radiation-enhancing effect, as indicated in previous studies [21,22]. The delay in growth of the secondary tumors and appearance of fewer lung metastases in the NBTXR3 + HDXRT + LDXRT + ICIs mice suggest that this treatment induced a more potent systemic antitumor immune response than the HDXRT + LDXRT + ICIs strategy alone. We previously showed that giving 
NBTXR3 with XRT led to a significantly improved abscopal effect relative to XRT alone by increasing the number CD8 $T$ cells and augmenting activities of various antitumor immune pathways [11]. In the current study, both NanoString and flow cytometry analyses of the secondary tumors confirmed that integrating NBTXR3 into dualdose (HDXRT + LDXRT) radiation promoted the activities of major immune pathways, elevated the expression of a wide range of antitumor genes, increased the percentage of CD8 $\mathrm{T}$ cells, and increased the CD8 T cell/ Treg ratio. CD8 $\mathrm{T}$ cells are known to have vital roles in antitumor immunity and thus having higher numbers of CD8 T cells would presumably enhance antitumor capacity [23]. Having higher CD8/Treg ratios has also been reported to correlate with better treatment outcomes [24, 25]. Collectively, these changes suggest that the addition of NBTXR3 created an immune environment that favors cancer killing. Moreover, because Tregs act as suppressor cells in terms of mounting an antitumor immune response [26, 27], the lower density of Tregs and higher CD8/Treg ratios in the NBTXR3 + HDXRT + LDXRT + ICIs mice relative to the NBTXR3 + HDXRT + ICIs mice suggest that LDXRT may reduce immune suppression by downregulating Tregs. This would be consistent with our previous finding that low-dose radiation (i.e., two 1-Gy fractions) could promote an antitumor immune response by reprogramming the immune microenvironment [7].

We studied the TCR repertoire of TILs from the metastatic sites to determine how differences in their amino acid sequences could explain the improved anti-tumoral effects that is observed with NBTXR3. We discovered that the two NBTXR3-treated mice subgroups shared more overlapping TCR $\beta$ sequences within each group compared to those shared within the corresponding nonNBTXR3 mice subgroups. These increases were significant only among the abscopally treated tumors (i.e., mice that received only high-dose radiation). This finding suggests that NBTXR3 might be inducing the expression of specific tumor epitopes that are recognized by primary site TILs with conserved TCRs that control metastatic disease after migration to those sites. The absence of significantly higher overlap frequencies with NBTXR3 among the high-and-low dose irradiated (Radscopal ${ }^{\mathrm{TM}}$ ) mice could be due to the generation of different TILs within the metastatic sites that are specific to epitopes induced by the low dose radiation. Overall, our TCR studies indicate that NBTXR3 reshapes the T cell repertoire and that might contribute to the improved antitumoral response with NBTXR3.

Relapses of lung cancer are common [28, 29], and thus the ability to evoke robust, long-term immune memory against recurring cancer cells would be considerably useful. The prevention of tumor growth in the surviving mice in the NBTXR3 + HDXRT + LDXRT + ICIs group after tumor rechallenge illustrates that these mice may have acquired an effective and systemic antitumor immune memory status. Indeed, our flow cytometry findings confirmed that NBTXR3 + HDXRT + LDXRT + ICIs led to significantly higher percentages of effector memory CD4 $\mathrm{T}$ cells in the blood and central memory $\mathrm{CD} 4 \mathrm{~T}$ cells in the spleen. Most likely, the memory CD4 $\mathrm{T}$ cells initiated the memory immune response against the re-challenged tumors by facilitating activation and proliferation of CD8 T cells [30]. This hypothesis is supported by our discovery of higher percentages of CD8 $\mathrm{T}$ cells in both blood and spleen of the surviving mice. Logically, one might conclude that the memory CD4 T cells are responsible for effectively activating naïve CD8 $\mathrm{T}$ cells in response to the tumor rechallenge.

\section{Conclusion}

In summary, we found that integrating NBTXR3 nanoparticle into a high-dose-plus-low-dose radiation strategy slowed the growth of both primary and secondary tumors, suppressed the appearance of lung metastases, and increased survival rates. NBTXR3 also enhanced the activities of major antitumor immune signaling pathways and increased the CD8/Treg ratio in the secondary tumors. Moreover, mice treated with NBTXR3 + HDXRT + LDXRT + ICIs maintained potent, long-term antitumor immune memory that suppressed the growth of rechallenged tumors. This combination of NBTXR3, high-dose plus low-dose radiation, and immune checkpoint blockade may well improve treatment outcomes in metastatic lung cancer and reduce the side effects of systemic high-dose radiation in the clinic. We are currently in the process of testing whether this combination treatment strategy is effective in cancer patients at MD Anderson Cancer Center (Clinical trial. gov NCT03589339).

\section{Supplementary Information}

The online version contains supplementary material available at https://doi. org/10.1186/s12951-021-01163-1.

Additional file 1: Figure S1. Treatment outcomes after therapy with NBTXR3, high- and low-dose radiotherapy. A Changes in primary tumor volumes over time. B Changes in secondary tumor volumes over time. Mice were subcutaneously inoculated with $5 \times 10^{4} 344$ SQR cells in the right legs on day 0 (to establish primary tumors) and in the left legs on day 4 (to establish secondary tumors). NBTXR3 was delivered to the primary tumor by intratumoral injection on day 7 . Primary tumors were treated with three 12-Gy fractions on day 8, 9, and 10 (HDXRT). Secondary tumors were irradiated with two 1-Gy fractions on day 13 and 14 (LDXRT).

Additional file 2: Figure S2. Normalized Shannon clonality comparison of CDR3 $\beta$ and CDR3a clonotypes between NBTXR3-treated mice and their 
corresponding non-NBTXR3 groups. $P<0.05$ from Mann-Whitney $U$ tests was considered statistically significant. NS not significant.

\section{Acknowledgements}

We thank Christine F. Wogan, MS, ELS, from the MD Anderson Division of Radiation Oncology, for editorial contributions to several versions of this manuscript.

\section{Authors' contributions}

YH, SP, MAC, and JW designed the study. YH, HB, MW performed the experiments. YH, SP, COA analyzed the data. YH, SP, and COA wrote the manuscript. All of the authors discussed the results and reviewed the manuscript. All authors read and approved the final manuscript.

\section{Funding}

This work was supported by Cancer Center Support (Core) Grant CA016672 to The University of Texas MD Anderson Cancer Center; the Goodwin family research fund; the family of M. Adnan Hamed and the Orr Family Foundation to MD Anderson Cancer Center's Thoracic Radiation Oncology program; an MD Anderson Knowledge Gap award; Nanobiotix.

\section{Availability of data and materials}

The data and materials that support the findings of this study are available from the corresponding author, upon reasonable request.

\section{Declarations}

\section{Ethics approval and consent to participate}

All animal procedures were approved by the Institutional Animal Care and Use Committee at MD Anderson Cancer Center.

\section{Consent for publication}

We understand that the text and any pictures published in the article will be freely available on the internet and may be seen by the general public. The pictures and text may also appear on other websites or in print, may be translated into other languages or used for commercial purposes.

\section{Competing interests}

JW receives research support from GlaxoSmithKline, Bristol-Meyers Squibb, Merck, Nanobiotix, Mavu Pharmaceuticals, and Checkmate Pharmaceuticals; serves on the scientific advisory board for RefleXion Medical, MolecularMatch, OncoResponse, CheckMate, Mavu Pharmaceuticals, and Alpine Immune Sciences; is cofounder of Helios Oncology, MolecularMatch, and OncoResponse; is an advisor to AstraZeneca, Merck, MolecularMatch, Incyte, Aileron, and Nanobiotix; and holds patents for MP470 (amuvatinib), MRX34 regulation of PDL1, and RadScopal, an XRT technique to overcome immune resistance (MD Anderson Cancer Center has a trademark for RadScopal). SP is an employee of Nanobiotix.

\section{Author details}

'Department of Radiation Oncology, The University of Texas MD Anderson Cancer Center, 6565 MD Anderson Boulevard, Houston, TX 77030, USA. ${ }^{2}$ Department of Translational Science, Nanobiotix, Paris, France. ${ }^{3}$ Department of Radiation Oncology, Shandong Cancer Hospital and Institute, Shandong First Medical University and Shandong Academy of Medical Sciences, Jinan, China. ${ }^{4}$ Department of Radiation Oncology, Koc University School of Medicine, Istanbul, Turkey.

Received: 15 October 2021 Accepted: 23 November 2021 Published online: 11 December 2021

\section{References}

1. Vachani A, Sequist LV, Spira A. AJRCCM: 100-year anniversary. The shifting landscape for lung cancer: past, present, and future. Am J Respir Crit Care Med. 2017;195(9):1150-60.
2. US National Institute of Health $\mathrm{NCl}$. SEER cancer statistics review, 1975-2015. Bethesda: NCl; 2015.

3. Blandin Knight S, Crosbie PA, Balata H, Chudziak J, Hussell T, Dive C. Progress and prospects of early detection in lung cancer. Open Biol. 2017. https://doi.org/10.1098/rsob.170070.

4. Buchwald ZS, Wynne J, Nasti TH, Zhu S, Mourad WF, Yan W, Gupta S, Khleif SN, Khan MK. Radiation, immune checkpoint blockade and the abscopal effect: a critical review on timing, dose and fractionation. Front Oncol. 2018:8:612.

5. Badiyan SN, Roach MC, Chuong MD, Rice SR, Onyeuku NE, Remick J, Chilukuri S, Glass E, Mohindra P, Simone CB 2nd. Combining immunotherapy with radiation therapy in thoracic oncology. J Thorac Dis. 2018;10(Suppl 21):S2492-507.

6. Daly ME, Monjazeb AM, Kelly K. Clinical trials integrating immunotherapy and radiation for non-small-cell lung cancer. J Thorac Oncol. 2015;10(12):1685-93.

7. Barsoumian HB, Ramapriyan R, Younes Al, Caetano MS, Menon H, Comeaux NI, Cushman TR, Schoenhals JE, Cadena AP, Reilly TP, et al. Low-dose radiation treatment enhances systemic antitumor immune responses by overcoming the inhibitory stroma. J Immunother Cancer. 2020. https://doi.org/10.1136/jitc-2020-000537.

8. Bonvalot S, Le Pechoux C, De Baere T, Kantor G, Buy X, Stoeckle E, Terrier P, Sargos P, Coindre JM, Lassau N, et al. First-in-human study testing a new radioenhancer using nanoparticles (NBTXR3) activated by radiation therapy in patients with locally advanced soft tissue sarcomas. Clin Cancer Res. 2017;23(4):908-17.

9. Zhang P, Darmon A, Marill J, Mohamed Anesary N, Paris S. Radiotherapyactivated hafnium oxide nanoparticles produce abscopal effect in a mouse colorectal cancer model. Int J Nanomed. 2020;15:3843-50.

10. Marill J, Mohamed Anesary N, Paris S. DNA damage enhancement by radiotherapy-activated hafnium oxide nanoparticles improves cGASSTING pathway activation in human colorectal cancer cells. Radiother Oncol. 2019;141:262-6.

11. Hu Y, Paris S, Barsoumian H, Abana CO, He K, Wasley M, Younes Al, Masrorpour F, Chen D, Yang L, et al. Radiation therapy enhanced by NBTXR3 nanoparticles overcomes anti-PD1 resistance and evokes abscopal effects. Int J Radiat Oncol Biol Phys. 2021. https://doi.org/10.1016/j.jirobp. 2021.06.041.

12. Wang X, Schoenhals JE, Li A, Valdecanas DR, Ye H, Zang F, Tang C, Tang M, Liu CG, Liu X, et al. Suppression of type I IFN signaling in tumors mediates resistance to anti-PD-1 treatment that can be overcome by radiotherapy. Cancer Res. 2017;77(4):839-50.

13. Liu K, Zhao E, Ilyas G, Lalazar G, Lin Y, Haseeb M, Tanaka KE, Czaja MJ. Impaired macrophage autophagy increases the immune response in obese mice by promoting proinflammatory macrophage polarization. Autophagy. 2015;11(2):271-84.

14. Guo C, Yang XG, Wang F, Ma XY. IL-1alpha induces apoptosis and inhibits the osteoblast differentiation of MC3T3-E1 cells through the JNK and p38 MAPK pathways. Int J Mol Med. 2016;38(1):319-27.

15. Malik A, Kanneganti TD. Function and regulation of IL-1alpha in inflammatory diseases and cancer. Immunol Rev. 2018;281(1):124-37.

16. Dvorkin T, Song X, Argov S, White RM, Zoller M, Segal S, Dinarello CA, Voronov $\mathrm{E}$, Apte RN. Immune phenomena involved in the in vivo regression of fibrosarcoma cells expressing cell-associated IL-1alpha. J Leukoc Biol. 2006;80(1):96-106.

17. Shugay M, Bagaev DV, Turchaninova MA, Bolotin DA, Britanova OV, Putintseva EV, Pogorelyy MV, Nazarov VI, Zvyagin IV, Kirgizova VI, et al. VDJtools: unifying post-analysis of T cell receptor repertoires. PLoS Comput Biol. 2015;11(11):e1004503.

18. Heath WR, Miller JF. Expression of two alpha chains on the surface of T cells in T cell receptor transgenic mice. J Exp Med. 1993;178(5):1807-11.

19. Blumenthal GM, Bunn PA Jr, Chaft JE, McCoach CE, Perez EA, Scagliotti GV, Carbone DP, Aerts H, Aisner DL, Bergh J, et al. Current status and future perspectives on neoadjuvant therapy in lung cancer. J Thorac Oncol. 2018;13(12):1818-31.

20. Hendriks LEL, Henon C, Auclin E, Mezquita L, Ferrara R, Audigier-Valette C, Mazieres J, Lefebvre C, Rabeau A, Le Moulec S, et al. Outcome of patients with non-small cell lung cancer and brain metastases treated with checkpoint inhibitors. J Thorac Oncol. 2019;14(7):1244-54. 
21. Maggiorella L, Barouch G, Devaux C, Pottier A, Deutsch E, Bourhis J, Borghi E, Levy L. Nanoscale radiotherapy with hafnium oxide nanoparticles. Future Oncol. 2012;8(9):1167-81.

22. Bonvalot S, Rutkowski PL, Thariat J, Carrere S, Ducassou A, Sunyach MP, Agoston P, Hong A, Mervoyer A, Rastrelli M, et al. NBTXR3, a first-in-class radioenhancer hafnium oxide nanoparticle, plus radiotherapy versus radiotherapy alone in patients with locally advanced soft-tissue sarcoma (Act.In.Sarc): a multicentre, phase 2-3, randomised, controlled trial. Lancet Oncol. 2019;20(8):1148-59.

23. Durgeau A, Virk Y, Corgnac S, Mami-Chouaib F. Recent advances in targeting CD8 T-cell immunity for more effective cancer immunotherapy. Front Immunol. 2018;9:14.

24. Baras AS, Drake C, Liu JJ, Gandhi N, Kates M, Hoque MO, Meeker A, Hahn $\mathrm{N}$, Taube JM, Schoenberg MP, et al. The ratio of CD8 to Treg tumorinfiltrating lymphocytes is associated with response to cisplatin-based neoadjuvant chemotherapy in patients with muscle invasive urothelial carcinoma of the bladder. Oncoimmunology. 2016;5(5):e1134412.

25. Peng GL, Li L, Guo YW, Yu P, Yin XJ, Wang S, Liu CP. CD8(+) cytotoxic and FoxP3(+) regulatory T lymphocytes serve as prognostic factors in breast cancer. Am JTransl Res. 2019:11(8):5039-53.

26. Verma A, Mathur R, Farooque A, Kaul V, Gupta S, Dwarakanath BS. T-regulatory cells in tumor progression and therapy. Cancer Manag Res. 2019;11:10731-47.

27. Ohue Y, Nishikawa H. Regulatory T (Treg) cells in cancer: can Treg cells be a new therapeutic target? Cancer Sci. 2019;110(7):2080-9.

28. Comino-Mendez I, Turner N. Predicting relapse with circulating tumor DNA analysis in lung cancer. Cancer Discov. 2017;7(12):1368-70.

29. Gong J, Salgia R. Managing patients with relapsed small-cell lung cancer. J Oncol Pract. 2018;14(6):359-66.

30. MacLeod MK, Kappler JW, Marrack P. Memory CD4 T cells: generation, reactivation and re-assignment. Immunology. 2010;130(1):10-5.

\section{Publisher's Note}

Springer Nature remains neutral with regard to jurisdictional claims in published maps and institutional affiliations.

- fast, convenient online submission

- thorough peer review by experienced researchers in your field

- rapid publication on acceptance

- support for research data, including large and complex data types

- gold Open Access which fosters wider collaboration and increased citations

- maximum visibility for your research: over $100 \mathrm{M}$ website views per year

At BMC, research is always in progress.

Learn more biomedcentral.com/submissions 